\title{
Anúncios de medicamentos em revistas médicas: ajudando a promover a boa prescrição?
}

\author{
Drug advertisements in medical journals: \\ promoting good prescription?
}

José Augusto Cabral de Barros 1

Sabrina Joany 2

1 Departamento de Medicina Social, Centro de Ciências da Saúde, Universidade Federal de Pernambuco. Cidade Universitária, 50670-900 Recife PE. josebarros@uol.com.br 2 Mestranda do Curso de Mestrado em Saúde Coletiva, CCS/UFPE.
Abstract Supply and demand of drugs are influenced by different ways. Manufacturers' promotional strategies are outstanding a particular role being ascribed to advertising (ads) in medical journals. Considering that studies on this issue are not available in Brazil a research was performed aimed at evaluating ads using as parameters WHO criteria for drug information that should be included in any informative material. All advertisements published in Jornal de Pediatria, Revista Brasileira de Medicina and Jornal Brasileiro de Medicina issues from August/2000 up to February/2001 were evaluated verifying the presence of generic name, mechanism of action, pharmacologic effects, indications, contraindications, dosing information, adverse effects, drugs interactions, overdose, types of formulation marketed, manufacturer and/or importer. In the total of 1.774 pages, 539 were occupied by 649 ads. No one of referred criteria was found in all ads and only $20 \%$ include contraindications, adverse effects and drugs interactions. The most advertised drugs were antibiotics, antihipertensives and combination products. Instead of subsidize prescription and adequate drug utilization ads are clearly biased obeying to marketing goals since meaningful data are missing.

Key words Drugs advertising, Pharmaceutical industry, Drugs prescription
Resumo Ante a inexistência de estudos no Brasil, este trabalho se propôs a fazer uma avaliação dos anúncios com base nos critérios sugeridos pela OMS e que deveriam incluir-se em qualquer material de divulgação. Foram avaliados todos os anúncios contidos nas edições de agosto/2000 a fevereiro/2001 do Jornal de Pediatria, Revista Brasileira de Medicina $e$ Jornal Brasileiro de Medicina, averiguando a presença nos mesmos de: nome genérico, mecanismos de ação, efeito farmacológico, indicação, contra-indicação, posologia, reações adversas, interações, superdosagem, apresentação e fabricante/importador. Em um total de 1.774 páginas, 539 (30,4\%) se destinavam a anúncios, compreendendo 649 ao todo. Nenhum dos critérios propostos pela OMS esteve presente em todas as propagandas, e apenas em cerca de 20\% delas há referência a reações adversas, contra-indicações e interações. Os produtos mais anunciados foram antibióticos, hipotensores e os à base da associação de vários fármacos. Omitindo dados importantes, em especial contra-indicações, reações adversas e interações, os anúncios são tendenciosos, atendendo a propósitos mercadológicos, não sendo um meio que subsidie a prescrição e a utilização correta e segura dos produtos anunciados. Palavras-chave Propaganda de medicamentos, Indústria farmacêutica, Prescrição de medicamentos 


\section{Introdução}

Com o incremento da quimiossíntese industrial farmacêutica, nas últimas seis décadas, os medicamentos passaram a ocupar, de forma crescente, lugar de destaque e hegemônico como alternativa para a cura das doenças e alívio dos sintomas. A despeito dos inegáveis ganhos terapêuticos obtidos, os produtos farmacêuticos passaram a sofrer um uso indiscriminado e irracional, sobretudo em virtude da lógica de mercado que tudo transforma em mercadoria, privilegiando uma visão da saúde como fenômeno estritamente biológico, assim como o bem-estar e a felicidade, de forma mais ampla, como dependentes do consumo de variados bens e serviços (Barros, 1984).

Esse uso indiscriminado, obviamente, termina por acarretar conseqüências negativas importantes, tanto nos custos de ordem econômica que governos e indivíduos passaram a fazer de forma crescente, como nos malefícios no âmbito sanitário, traduzidos, particularmente, pelo aumento de efeitos colaterais ou reações adversas, por vezes bastante graves. Tais fenômenos, ao se intensificarem, provocaram, em diferentes países, em especial nos mais desenvolvidos, o estabelecimento de políticas de controles sobre os diferentes elos que integram a cadeia que vai da produção ao consumo, passando pela prescrição, dispensação, comercialização e uso final dos insumos farmacêuticos. Destacam-se tanto os controles sobre preços e margem de lucros, como sobre a busca do máximo de evidências da eficácia terapêutica e segurança, neste último caso, não só quando do registro e autorização iniciais (papel dos órgãos de vigilância sanitária), mas igualmente pelos sistemas de monitorização após a comercialização, de que se encarrega a farmacovigilância.

Entre os múltiplos fatores que já vêm sendo estudados como exercendo influência sobre a prescrição médica, destacam-se os relacionados às fontes de informação de que lançam mão os médicos, especialmente as produzidas e disseminadas pelos produtores. Como exemplos desses estudos poderiam ser citados os de Hemminki (1988) e Barros (1995). Neste último, realizado em Recife, apenas $21 \%$ dos médicos entrevistados manifestaram concordância quanto ao caráter desnecessário da atuação dos propagandistas. Quase a metade dos entrevistados declarou que os profissionais mencionados somente seriam dispensáveis caso estivessem asseguradas alternativas que substituíssem o pa- pel a eles atribuído (fontes de atualização e de amostras grátis; para alguns médicos, os propagandistas foram tidos "como um mal necessário"). No caso do estudo mencionado, feito por Hemminki, ficou evidenciado o papel prioritário dos propagandistas como sendo o de "agentes de vendas". Nas suas atividades promocionais junto aos médicos, os produtores de medicamentos utilizam tanto mecanismos diretos, como indiretos. Estes últimos contemplam o financiamento de programas chamados de "educação continuada", das revistas médicas ou de associações profissionais; relacionamento especial com autoridades sanitárias ou com médicos denominados key-physicians ou opinion-makers, a exemplo de especialistas famosos ou professores universitários. Classificam-se como instrumentos ou estratégias diretas a distribuição de amostras grátis, anúncios em revistas médicas, distribuição dos mais variados brindes, propagandistas, etc.

Trabalho publicado em 1993 avaliando anúncios em revistas médicas incluía o Brasil, ao lado de 17 outros países. O estudo pretendeu averiguar o grau de adesão dos mesmos aos critérios éticos na promoção dos medicamentos, propostos pela OMS. Aprovados em 1968 e revistos na assembléia da Organização em 1988, esses critérios visam orientar a promoção dos medicamentos de tal forma que os direitos dos consumidores sejam assegurados. $\mathrm{O}$ estudo concluiu que, na metade dos anúncios analisados, não constavam advertências e precauções e em $40 \%$ deles não foram incluídas alusões a contra-indicações, nem reações adversas (Herxheimer $e t$ al., 1993). Já em relação ao inquérito realizado por Barros, tomando os mesmos parâmetros do presente estudo como referência (critérios sugeridos pela OMS como fundamentais na informação para profissionais que lidam com medicamentos, tal como se explicita mais adiante), o referencial foi distinto. Manuais de uso habitual por prescritores no Brasil e nos Estados Unidos foram comparados, na busca de discrepâncias no teor do que é informado para os mesmos produtos nos dois países. Foram detectadas diferenças importantes nas informações brindadas aos profissionais, concentradas, precisamente, nas contra-indicações, reações adversas e interações, representando esses achados mais um exemplo de padrão distinto de conduta das empresas em diferentes países (Barros, 2000). Esse duplo padrão, aliás, foi constatado também com respeito a anúncios estudados nas edições inglesa e indiana do British $\mathrm{Me}$ - 
dical Journal. Enquanto na primeira 20\% dos anúncios foram tidos como cientificamente inadequados, na segunda foram apenas em 3\% dos casos (Gitanjali et al., 1997). De igual forma, em 141 anúncios publicados em revistas africanas de países francófonos, em apenas 41 constatouse a adesão a padrões franceses de objetividade e exatidão. "Reações adversas" e "contra-indicações" não foram referidas, respectivamente, em 37 e 30 dos anúncios e, ainda quanto às primeiras, as informações estavam incompletas em 20 anúncios. Já as “indicações” estavam ausentes em 5 e exageradas em 42 das inserções publicitárias (Chirac et al., 1993).

$\mathrm{Na}$ Espanha foi realizado estudo a respeito dos componentes publicitários e farmacológicos de anúncios publicados em quatro revistas. Entre outras conclusões, foi constatado que a proporção do conteúdo publicitário era três vezes maior que o farmacológico (Valpuesta et al., 1993).

\section{Procedimentos metodológicos}

Foram selecionadas três das principais revistas médicas brasileiras - Jornal de Pediatria, Revista Brasileira de Medicina e Jornal Brasileiro de Medicina $(J B M)$-, tendo-se procedido, nas edições publicadas em 2000/2001, em todos os anúncios inseridos nos números selecionados, à verificação da presença ou não dos critérios básicos propostos pela OMS como obrigatórios em todo e qualquer material que venha a ser distribuído a profissionais de saúde. Esses critérios são: "nome genérico", "mecanismos de ação", "efeito farmacológico", "indicação terapêutica", "contra-indicações”, "posologia”, "reações adversas”, "interação medicamentosa”, "superdosagem", "apresentação farmacêutica" e "fabricante/importador". Em seguida, com base nas categorias terapêuticas mais anunciadas foram selecionados, aleatoriamente, para uma análise crítica mais acurada, anúncios de produtos relacionados às mencionadas categorias. Nessa revisão, fez-se uso de As bases farmacológicas da terapêutica (Goodman \& Gilman, 1996) e do Dicionário terapêutico (Korokolvas, 1997).

\section{Resultados}

Em uma primeira etapa do estudo, foi possível traçar um perfil dos anúncios veiculados nas revistas selecionadas. Entre as 1.774 páginas que compunham as edições selecionadas, 539 (30\%) eram ocupadas por anúncios. No total, foram encontrados 649 anúncios, dos quais, $311(47,92 \%)$ ocupavam uma página completa, $268(41,29 \%)$ tomavam menos de uma página e 70 (10,79\%) ocupavam mais de uma página. A tabela 1 apresenta o conjunto dos critérios presentes no total de anúncios, salientando-se que apenas quatro deles aparecem em mais da metade dos anúncios: "nome genérico", "fabricante/importador", "apresentação farmacêutica" e "indicação terapêutica". Vale ressaltar que nenhum dos anúncios continha todos os critérios.

Apenas em cerca de $20 \%$ dos anúncios há referência a reações adversas, contra-indicações e interações. Os grupos terapêuticos mais anunciados (Tabela 2) foram antibióticos, hipotensores e os produtos à base da associação de vários fármacos, seguidos dos antiinflamatórios não esteroidais (AINES) e dos hipno-sedantes.

As figuras 1, 2 e 3 evidenciam a ausência da informação sobre "reações adversas", "contraindicações" e "interações" na maior parte dos anúncios das três revistas avaliadas (no conjunto delas, conforme mostra a tabela 1); duas das informações figuravam tão somente em $20 \%$ dos anúncios, estando a "interação medicamentosa” presente em uma proporção ainda menor $(16 \%)$.

No estudo do conteúdo dos anúncios, direcionado à detecção das informações farmacológicas que, pela sua importância, deveriam estar

Tabela 1

Freqüência dos critérios sugeridos pela OMS para constarem das informações sobre medicamentos presentes no conjunto das três revistas selecionadas (em números absolutos e em percentagem).

\begin{tabular}{lrr}
\hline $\begin{array}{l}\text { Critérios propostos } \\
\text { pela OMS }\end{array}$ & Anúncios com presença & \% \\
\hline Nome genérico & 632 & 97,38 \\
Mecanismos de ação & 137 & 21,11 \\
Efeito farmacológico & 58 & 8,97 \\
Indicação terapêutica & 384 & 59,17 \\
Contra-indicações & 128 & 20,00 \\
Posologia & 294 & 45,30 \\
Reações adversas & 130 & 20,00 \\
Interação medicamentosa & 107 & 16,00 \\
Superdosagem & 67 & 10,00 \\
Apresentação farmacêutica & 508 & 78,27 \\
Fabricante/importador & 629 & 96,92 \\
Total & $\mathbf{6 4 9}$ & $\mathbf{1 0 0 , 0}$ \\
\hline
\end{tabular}




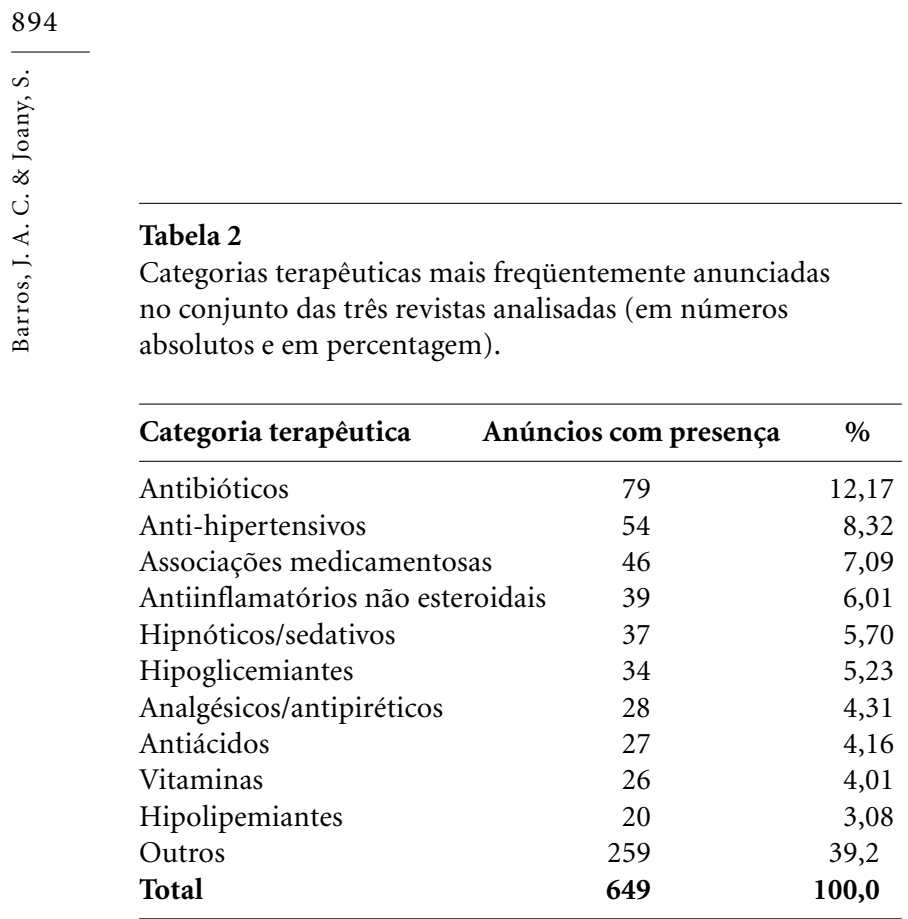

presentes, efetuou-se uma avaliação de 39 anúncios, dos quais 19 se referiam a AINES, 12 a anti-hipertensivos e 9 a antibióticos. No primeiro caso, 16 anúncios não faziam alusão a interações e em apenas 4 e 5 deles estiveram contempladas, respectivamente, referências a contra-indicações e reações adversas. Neste último caso, o Celebra ${ }^{\circledR}$ (celecoxib) alude a reações mais comuns (de natureza gastrointestinal), mas não refere várias outras, a exemplo de broncoespasmo, vertigem, urticária, prurido, epigastralgia. Chama a atenção o fato de que em sete casos, nenhuma das informações ora consideradas estava presente, o que se observa em 2 de 3 anúncios do diclofenaco (Voltaren ${ }^{\circledR}$ e Artren $®$ ). Essa ausência se dava, igualmente, em 5 dos 8 anúncios de antibióticos, (informações presentes apenas nos casos do Amoxil ${ }^{\circledR}$ (amoxicilina), Clavulin ${ }^{\circledR}$ (amoxicilina e clavulinato) e Tavanic ${ }^{\circledR}$ (levofloxacina); já com respeito aos anti-hipertensivos, em apenas um dos 12 anúncios avaliados nessa etapa, as 3 informações consideradas estavam presentes. Entretanto, com respeito ao Tavanic $500 \AA$ (levofloxacina), foram apresentadas como reações adversas apenas as mais freqüentes (diarréia, náusea, vaginite e elevação das taxas de enzimas hepáticas), desconsiderando potenciais reações igualmente importantes como dores abdominais, prurido, rash cutâneo, insônia, visão turva, insuficiência renal aguda, artrite, confusão mental, convulsões, depressão, granulocitopenia, alucinações, hipoglicemia, pancreatite, paranóia, fotossensibilidade, colite pseudomembranosa, rabdomiólise, tendinite, trombocitopenia, choque anafilático, eritema multiforme.

Não havia nenhuma alusão às contra-indicações do Klaricid (claritromicina) em pacientes com hipersensibilidade conhecida aos antibióticos macrólidos. Esse antibiótico também está contra-indicado em pacientes com problemas cardíacos preexistentes que façam uso de terfenadina (arritmia, bradicardia, prolongamento do intervalo QT, cardiopatia isquêmica, insuficiência cardíaca congestiva) ou que tenham distúrbios eletrolíticos.

Quanto ao anti-hipertensivo Micardis ${ }^{\circledR}$ (telmisartan), o anúncio não referia o fato de que o tratamento simultâneo com outros fármacos que atuam sobre o sistema renina-angiotensina-aldosterona esteve associado com hipotensão aguda, hiperazotemia, oligúria e, raramente, insuficiência renal. Não há, igualmente, referência à contra-indicação do produto nos casos de gravidez, amamentação e presença de insuficiência hepática ou renal severas.

\section{Discussão}

Habitualmente os médicos não admitem que sua atividade prescritora sofre influência, em maior ou menor grau, das atividades promocionais de que lança mão a indústria farmacêutica, nas quais são investidos recursos substanciais. Particularmente dirigidas aos médicos, tais despesas são, ao final, custeadas pelo consumidor, pois estão embutidas no preço dos produtos. Dispêndios tão significativos (da ordem de 20 a $25 \%$ do faturamento global) realizados com propaganda só podem explicar-se no fato de que há o esperado retorno em termos de vendas e lucro. Em 1998, nos EUA, a indústria gastou 10,8 bilhões de dólares na referida atividade (74,8 milhões foram aplicados para fazer lobby junto ao Governo) (Silverstein, 2000).

A publicidade inserida nas revistas médicas, responsável pelo custeio das mesmas, já foi objeto de interesse de diferentes estudos, fora do Brasil. Um deles procurou mostrar a relação existente entre o aumento da prescrição de determinado produto e o número de anúncios veiculados (Krupka \& Vener, 1985). Por outro lado, já se constatou que uma atitude pouco crítica dos médicos diante das estratégias promocionais das empresas farmacêuticas está associada a uma prescrição de baixa qualidade (HaaijerRuskamp \& Hemminki, 1993). Um inquérito feito em Boston (EUA) sobre hábitos de prescrição entrevistou 85 médicos, $68 \%$ dos quais opinaram que os anúncios tinham uma influência 
Figura1

Porcentagem da informação sobre contra-indicações nas três revistas estudadas.

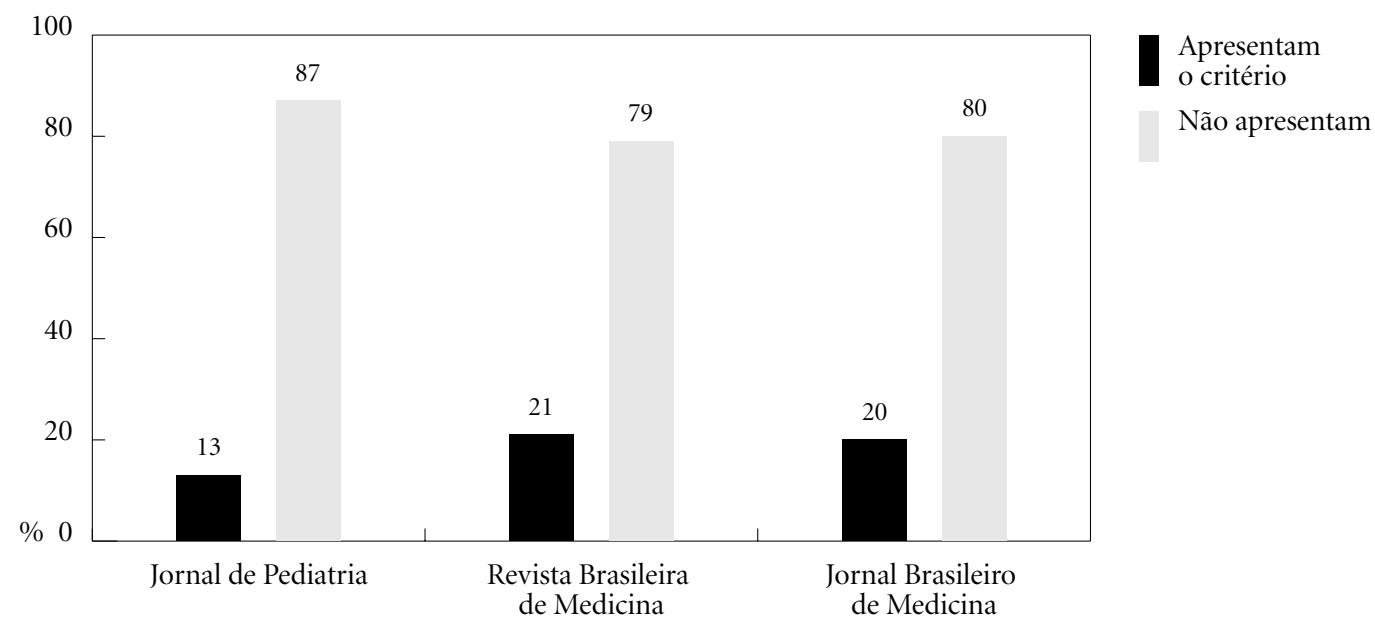

“mínima”, 28\% contestaram que essa influência era "moderada" e apenas 3\% dos entrevistados consideraram "grande" a influência em questão. A maioria classificou os artigos científicos sobre medicamentos como tendo influência "importante" ou "moderadamente importante". Entretanto, quando perguntados sobre ativadores ou vasodilatadores cerebrais sem real eficácia terapêutica, bastante anunciados em revistas médicas pouco antes do estudo, os mesmos entrevistados os consideraram úteis para pacientes idosos portadores de quadro clínico de confusão mental.

Dois médicos e um farmacêutico se encarregaram de avaliar uma amostra de 109 anúncios publicados em 10 grandes revistas dos EUA. Além do valor educativo e conteúdo global dos anúncios, pretendeu-se avaliar sua adequação às normas estabelecidas pela Food and Drug Administration (FDA), órgão responsável, nos Estados Unidos, pelo controle sobre todos os elos da cadeia de produção, dispensação, comercialização e consumo de medicamentos e alimentos. Dos 109 anúncios, 92\% cometiam infrações a pelo menos uma dessas normas. Os problemas principais constatados incluíam afirmações imprecisas, informação inadequada ou incompleta sobre efeitos adversos, contra-indicações, eficácia e segurança dos produtos anunciados (Wilkes et al., 1992). Nossos achados, em grande medida, coincidem com os de Wilkes et al., uma vez que, nos anúncios por nós analisados, não se incluíam informações que, como propõe a OMS, deveriam estar presentes rotineiramente em qualquer material de divulgação distribuído a prescritores e a outros profissionais que lidem com medicamentos. Em $80 \%$ dos anúncios, por exemplo, não constavam as informações mencionadas, o que é absolutamente injustificável, pressupondo-se que o propósito da publicidade veiculada seria disponibilizar aos profissionais que lêem ou consultem as revistas informações básicas e confiáveis sobre o produto. As lacunas observadas se referiam, no caso, a dados que são imprescindíveis para o uso correto e, sobretudo, mais seguro dos produtos em questão.

Avaliação mais recente, feita com base em 207 anúncios de cinco revistas médicas da Rússia, constatou que, em apenas $11 \%$ dos casos, reações adversas e advertências quanto à segurança eram abordadas; proporção ainda menor (5\%) fazia alusão às interações, aparecendo o nome genérico em $39 \%$ dos anúncios. Os autores chamam a atenção para o fato de que o comportamento das empresas detectado vem sendo documentado, nas duas últimas décadas, em diferentes países subdesenvolvidos, mas também nos desenvolvidos onde vigoram controles mais estritos. Entre as possíveis explicações para a persistência do fenômeno, os autores apontam: não preparação dos médicos para avaliarem os 
Figura 2

Porcentagem da informação sobre reações adversas nas três revistas estudadas.

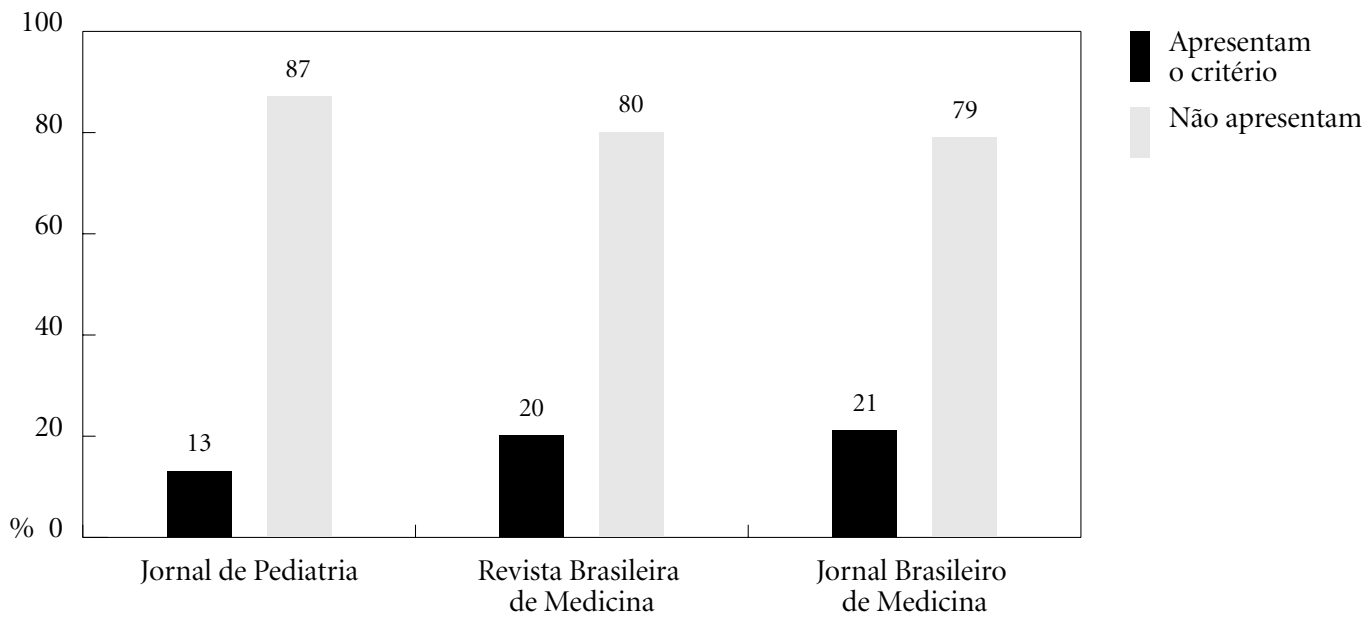

Figura 3

Porcentagem da informação sobre interações medicamentosas nas três revistas estudadas.

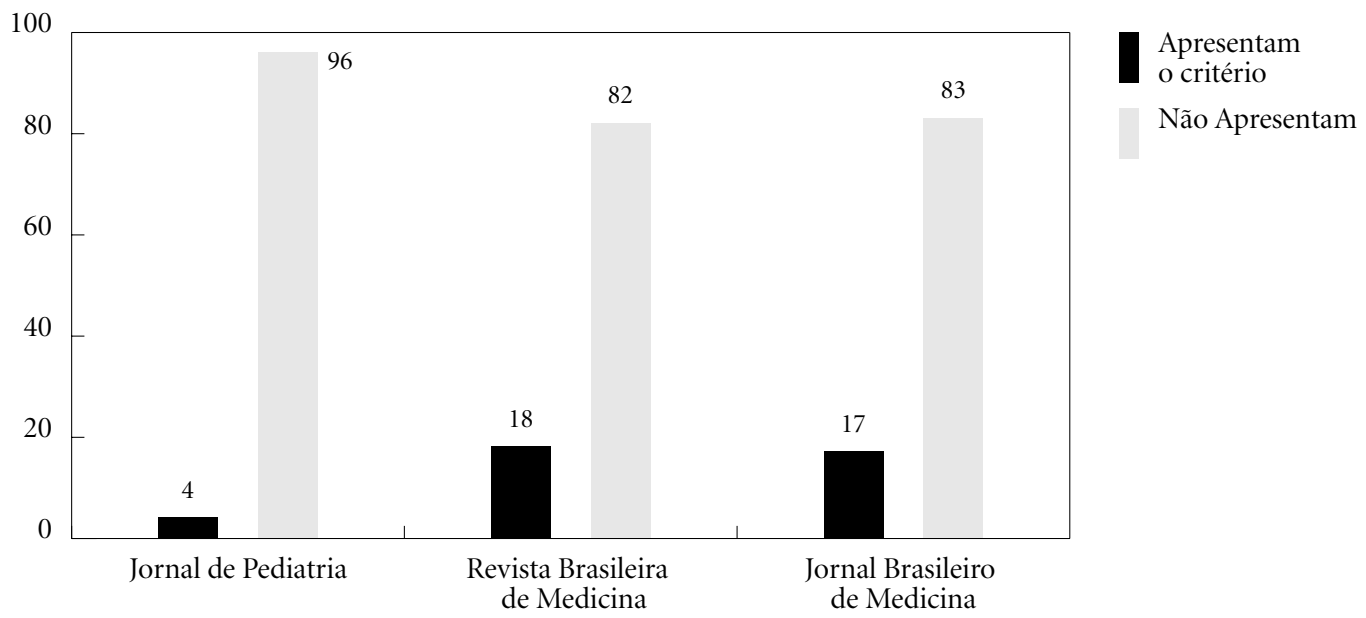

anúncios, deixando de reconhecer problemas existentes na maior parte deles; as sanções para os abusos são incapazes de deter as empresas (Vlassov et al., 2001).

No presente estudo, os produtos à base de associações de vários princípios ativos estão no terceiro posto, totalizando quase $30 \%$ dos anúncios, precedidos apenas pelos antibióticos e anti-hipertensivos, que, junto com os hipnóticos, compõem as categorias terapêuticas mais anunciadas. Vale recordar que a combinação de diferentes princípios ativos em um único produto não deve ser a opção preferencial na farmacoterapia, considerando-se a elevação de custos e a possibilidade de incremento nos riscos de efeitos adversos ante a somação potencial dos mesmos. A justiticativa, em termos farmacológicos, para a opção por produtos combinados restrin- 
ge-se às ocasiões em que existe sinergismo (eficácia terapêutica maior na associação do que com o uso dos fármacos isolados) ou facilidades importantes quando da utilização pelos pacientes.

Os anúncios de produtos vitamínicos apareceram em lugar importante, em especial na revista orientada aos pediatras (quarto lugar entre os mais freqüentes). Estudos realizados dão conta da importância outorgada aos polivitamínicos, consumidos largamente, seja a partir de presrições médicas, seja indicados por balconistas ou por decisão mais ou menos direta do próprio usuário. Inquérito feito em Recife, em amostra representativa das farmácias então existentes, evidenciou a presença de polivitamínicos como o primeiro entre os produtos mais indicados por balconistas, a eles se seguindo os antidiarréicos e os antibióticos (Barros, 1997). Há uma crença generalizada na energia, saúde, vitalidade que as vitaminas poderiam brindar (Barros, 1995), tendo-se até ampliado as condições para as quais se pensa terem as vitaminas uma ação terapêutica eficaz (Chetley, 1995).

Revisão farmacológica efetuada evidenciou que em muitos dos anúncios de produtos mais freqüentemente incluídos nas revistas, à base de AINES, anti-hipertensivos e antibióticos, havia lacunas importantes com respeito a reações adversas, contra-indicações e interações.

Já é consensual a idéia de que se fazem necessários controles sobre as práticas promocionais da indústria farmacêutica. Os códigos de auto-regulamentação por ela formulado, que contemplam normas que deveriam ser observadas em relação aos anúncios, já teve sua ineficácia sobejamente demonstrada, até mesmo pela ausência ou debilidade das sanções quando das infrações cometidas, o que se constitui, de fato, não uma exceção, mas a regra (Lexchin, 1988, 1994; Herxheimer \& Collier, 1990; HAI, 1994). $\mathrm{Na}$ revisão por nós efetuada deste tema, apenas no caso da avaliação de 141 anúncios feita na Austrália, em anos diferentes, foi constatada uma melhora quanto às infrações ao Código de Conduta da Australian Pharmaceutical Manufacturers Association relativas a alegações publicitárias inaceitáveis. Já no que respeita a infrações de natureza técnica, detectou-se um incre- mento nas violações, passando de 29 para $40 \%$, entre 1989 e 1992 (Carandang \& Moulds, 1994).

\section{Conclusões}

As conclusões principais a que se chegou no presente estudo podem ser, assim, sintetizadas: - A veiculação de anúncios nas revistas médicas estudadas - e pode-se pressupor que esse comportamento se repete nas revistas médicas em geral - ocupa um número significativo de páginas.

- Há lacunas importantes, nos anúncios veiculados, quanto a informações básicas para orientar a prescrição e que deveriam constar, obrigatoriamente, dos mesmos, se partimos do pressuposto de que o propósito da publicidade destinada aos médicos é possibilitar-lhes acesso aos dados técnico-farmacológicos dos produtos anunciados.

- Sendo a indústria farmacêutica uma fonte hegemônica de informação sobre medicamentos, ante a inexistência de fontes alternativas independentes, pode-se inferir que o uso dos produtos farmacêuticos podem estar tendo uma orientação inadequada, seja na sua prescrição, seja na sua dispensação e comercialização, extrapolando suas indicações técnicas para atender interesses que não propriamente terapêuticos.

- É urgente a necessidade de que autoridades sanitárias e da área da educação, somadas a iniciativas das associações profissionais, criem instrumentos de atualização e divulgação no campo da farmacoterapia que guardem independência do financiamento dos produtores e propiciem informação com características técnicas e científicas. Cabe, ainda, à autoridade sanitária implementar legislação já existente que pode minimizar os malefícios das estratégias promocionais das empresas farmacêuticas dirigidas seja aos profissionais de saúde, seja ao grande público. Na publicidade ou promoção de produtos que requerem prescrição médica, a ser dirigida exclusivamente aos médicos, a Resolução 101 da Anvisa (Agência Nacional de Vigilância Sanitária) de 30.11.2001 impõe a inclusão dos tópicos objeto da avaliação que se constituiu no substrato do presente artigo. 


\section{Referências bibliográficas}

Barros JAC 1984. Medicalización y Salud. Cuadernos Médico-Sociales 28:25-31.

Barros JAC 1995. Propaganda de medicamentos: atentado à saúde? Sociedade Brasileira de Vigilância de Medicamentos-Ed. Hucitec, São Paulo.

Barros JAC 1997. Balconistas de farmácia - ajudando a promover o uso racional de medicamentos? Jornal Brasileiro de Medicina 73(2):120-127.

Barros JAC 2000. A (des)informação sobre medicamentos: o duplo padrão de conduta da indústria farmacêutica. Cadernos de Saúde Pública 16(2):110-119.

Carandang ED \& Moulds RFW 1994. Pharmaceutical advertisements in Australian medical publications have they improved? The Medical Journal of Australia 161:671-672.

Chetley A 1995. Las vitaminas estimulan el crescimento de la industria farmacéutica, pp. 249-262. In: Medicamentos problema. Acción Internacional para la Salud, Chimbote.

Chirac P, Pikon A, Poinsignon Y \& Vitry A 1993. Drug marketing in French-speaking African countries. Social Sciences and Medicine 36:1.541-1.543.

Gitanjali B, Shash CH, Tripathi KD \& Sethuraman KR 1997. Are drug advertisements in India edition of BMJ unethical? British Medical Journal 315:459.

Goodman \& Gilman 1996 (9ạ ed.). As bases farmacológicas da terapêutica. Editora Guanabara Koogan, Rio de Janeiro.

HAI (Health Action International) 1994. Self regulation or self-deception? Commentary on the 1994 Revision of the IFPMA Code of Pharmaceutical Marketing Practices. HAI Europe, Amsterdam.

Haaijer-Ruskamp FM \& Hemminki E 1993. Social aspects of drug use, pp. 97-124. In Dukes MNG (ed.). Drug utilization studies. WHO Regional Publications, European Series № 45, Kopenhagen.

Hemminki E. 1988. Factors influencing prescribing, pp. 22-35. In Ghodse H \& Khan (ed.). Psychoactive drugs: improving prescribing practices. WHO, Genebra.
Herxheimer A, Lundborg CS \& Westrholm B 1993. Advertisements for medicines in leading medical journals in 18 countries: a 12-month survey of information content and standards. International Journal of Health Services 23(1):161-172.

Herxheimer A \& Collier J 1990. Promotion by the British pharmaceutical industry, 1983-1988: a critical analysis of self-regulation. British Medical Journal 300: 307-311.

Korokolvas A 1997. Dicionário Terapêutico Guanabara. Ed 1997/98. Editora Guanabara Koogan, Rio de Janeiro.

Krupka LR \& Vener AM 1985. Prescription drug advertising: trends and implications. Social Science \& Medicine 20(3):191-7.

Lexchin J 1988. Pharmaceutical promotion in New Zeland. Community Health Studies 12(3):264-272.

Lexchin J 1994. Canadian marketing codes: how well are they controlling pharmaceutical promotion? International Journal of Health Services 24(1):91-103.

Silverstein K 2000. Millions for Viagra, pennies for diseases of the poor - Research money goes to profitable lifestyle drugs (obtido em site da internet)

Valpuesta FJL, Alonso AH, Díaz JAV, Lópes JAF \& Alonso AF 1993. Análisis de la publicidad medicamentosa en revistas médicas no especializadas. Revista Clinica Española 192: 130-133.

Vlassov V, Mansfield P \& Vlassova A. 2001. Do drug advertisements in Russian medical journals provide essential information for safe prescribing? Western Journal of Medicine 174:391-394.

Wilkes MS, Doblin BH \& Shapiro MF 1992. Pharmaceutical advertisements in leading medical journal: experts' assessments. Annals of Internal Medicine 116: 912-919.

Artigo apresentado em 6/5/2002

Aprovado em 8/8/2002

Versão final apresentada em 11/10/2002 